\title{
Suspended liminality: breastfeeding and becoming a mother in two NICUs
}

\author{
Khulood Kayed Shattnawi \\ Jordan University of Science and Technology \\ E-mail:khuloods@just.edu.jo
}

\begin{abstract}
Objectives: This paper presents the results of an ethnographic study of breastfeeding and mothers' experiences within two neonatal intensive care units (NICU).

Methods: The data collection involved 135 hours of participant observation over a 6-month period and semi-structured interviews of 17 mothers.

Results: Data analysis showed that the mothers faced many obstacles that prohibited them from practicing breastfeeding within the NICUs, which impacted on their experiences as mothers. Their experiences were revealed as a developing process as their feeling changed from fearful and terrifying toward becoming and feeling like a 'real' mother. Four distinct themes emerged; the first highlighted the crisis, which involved the mother's feelings of emotional instability, their strategies for coping such as not visiting the baby, and recognition of the NICU as a stressful environment. The second theme described issues relating to control and power. This involved the perception of having a lack of control and needing to seek permission, the use of language as a mechanism for control, and mothers being placed in a subordinate role. The third theme related to the separation, which included difficulties of acceptance, feeling like stranger and not being important, and the need for physical closeness. The final theme, becoming a mother, included issues such as the special moments, breastfeeding as a turning point, and practical and informational needs.

Conclusions: A finding suggests that mothers, who deliver prematurely, may have their rite of passage into motherhood interrupted, resulting in them being placed in a position of suspended liminality.
\end{abstract}

Keywords: Breastfeeding; Preterm Infants; Mothers' Experiences; Neonatal Intensive Care Unit; Liminal; Jordan.

\section{Introduction}

"Breast is best" is a phrase well accepted by the heath care professions. However, despite the well documented benefits of breastfeeding, preterm infants cared for in neonatal intensive care units in Jordan are routinely given artificial feed and there are minimal strategies employed to promote or support breastfeeding. This study sought to identify the reasoning behind this situation and explore the possible consequences in two different neonatal intensive care units (NICU) in Irbid, Jordan.

Bottle-feeding is the norm in many Jordanian NICUs with a delay in introducing breastfeeding for infants until after they tolerate a certain amount of formula feed with no complications. The NICUs in Jordan often have a more child-centred care environment, where parents are treated as visitors. In many developed countries, however, the NICUs are designed to accommodate the new family-centred care approach, which promotes more family participation in the infant's care plan (Browne 2003) with the implication of the novel developmental care approach. These approaches are not routinely part of the NICUs in Jordanian hospitals.

Breastfeeding is a strategy that can be used to facilitate the mother-infant relationship and attachment process (Flacking et al. 2006). Kearvell and Grant (2010) reviewed the literature on how nurses can support the mother-infant attachment process in NICUs. They found that Kangaroo Care, breastfeeding and participating in daily routine care such as changing nappies, bathing, or changing positions were essential elements in promoting the mother-infant attachment, the mothers' feeling of closeness, the mothers' confidence, and the physiological and behavioural status of the infants. They also reported that providing mothers with psychological support and effective communication helped in facilitating mother-infant attachment, alleviating maternal anxiety and enhancing mothers' satisfaction and confidence.

Breastfeeding may give the mothers a level of satisfaction which contributes to their feeling of being a real mother. Mothers considered breastfeeding as an important part of being a good mother because it is a unique maternal act that no other person could provide (Lupton \& Fenwick 2001). Kavanaugh et al.(1997) indicated that providing milk was a rewarding experience for preterm infants' mothers as participating in caring for their infants helped them to cope with the emotional stress. However, many factors in the neonatal intensive care unit may affect breastfeeding including long-term maternal-infant separation, the infants' condition, delaying of milk expression and the strange environment (Hartmann \& Ramsay 2005).

Studies of women's experiences of breastfeeding in the neonatal units revealed that failure to breastfeed is associated with feelings of being a bad mother (Fenwick, et al. 2001), accompanied by feeling of grief, sorrow, guilt and dissatisfaction (Shakespeare et al. 2004, McInnes \& Chambers 2008). A 'good mother' is described by both nurses and mothers, as being physically available for her child, interested in gaining knowledge about the baby's 
condition and keen to care for the baby when possible (Lupton \& Fenwick 2001, p. 1019).

The transformation of a woman into a mother is a complex experience that includes both physical and emotional involvements (Redwood 2007). The ease of this transition depends not only on the quality of the birth experience, but also on the support provided for the mothers and the personal characteristics of the person involved (Redwood 2007). The challenge faced by the mothers of preterm infants in their transition may be different from those of full term infants, and may be affected by the context in which this transition occurs. It has been documented that mothers of preterm infants experience more difficulties in their transition to motherhood than mothers of full term infants (Shin \& White-Traut 2007). For mothers of preterm infants' the transition includes devastating feelings of fear, disappointment and anxiety because of uncertainties regarding their baby's survival (Bialoskurski, et al. 2002). Barclay et al. (1997) detected not only a biological but also an emotional and personal sense of becoming a mother. These mothers were unready to become a mother and they undergo reconstruction of self. Becoming a mother for these mothers made them feel isolated and depleted instead of feeling nurtured and supported.

Becoming a mother is a transformation process in which women move from one state to another. The transformation involves being "betwixt and between", in which a person often feels isolated or disconnected from the before and after states (Turner 1987). During this process, a phase of ambiguity and uncertainty occurs, which was described as being in liminal state (Van Gennep 1960). The use of liminality as a framework to understand breastfeeding experience was first introduced by Davis-Floyd and Sargent (1997) and further elaborated by others (Schmied \& Lupton 2001; Mahon-Daly \& Andrews 2002; Sachs 2005). However, there is only one study detected that identified liminal properties with parenting transitions in parents of very preterm infants (Watson 2011).

In seeking to explore mothers' experiences of breastfeeding in Jordanian NICUs, this study revealed a number of aspects relating to women's experiences of breastfeeding and becoming a mother during their infant stay in the NICUs that hold relevance to both Jordan and a more universal perspective. The concept of suspended liminality that will be introduced in this paper will builds on the small volume of literature around the concept of liminality and becoming a mother for mothers of preterm infants.

Breastfeeding and becoming a mother experiences within NICUs are an area of care not previously studied in depth in Jordan. As a result of this deficiency in literature, there was no detailed information on how mothers and health care workers perceive breastfeeding experiences, and how breastfeeding is connected to the process of becoming a mother in an Arabic country. The aim of this ethnographic study, therefore, was to explore the experiences and perceptions of mothers and health care staff in relation to breastfeeding and mothering preterm infants in two hospitals within Northern Jordan.

\section{Methodology and methods}

This research paper is part of a larger $\mathrm{PhD}$ study aimed at exploring breastfeeding practices and experiences in two different neonatal intensive care units in Jordan. As there is very little literature on breastfeeding and becoming a mother in NICU in Jordan, a qualitative research methodology was selected to understand and interpret what is happening within the neonatal intensive care units. The choice of adopting an ethnographic research methodology appeared appropriate for the study's aims because it offered a way of observing in detail social processes that take place within NICUs including breastfeeding activities, nursing interventions, parent participations and medical procedures. Our understanding of these issues will lead to designing accessible, comprehensive and effective breastfeeding strategies in Jordan's NICUs.
This study was based on fieldwork adopting the observer participant role, supported by formal and informal interviews. A research diary of personal reflections, emerging themes, and own observation and attitudes was maintained, which all form part of the data. The use of these methods provided a total picture of the phenomena of study and validated each other (Roper \& Shapira 2000). The data collection involved 135 hours of participant observation over a 6-month period and 32 semi-structured interviews of 17 mothers, 10 nurses, and 5 physicians. This paper presents the mothers' perspectives and experiences.

\subsection{Participant observation}

For the purpose of this study, the observer-as-participant role (Gold 1958) was adopted. This helped to give the researcher more time to interact with mothers and other health care professionals. In addition, as the researcher was a former clinical instructor nurse in the two units, she could freely move around both units without being intrusive, which allowed for spontaneous observation and access to opportunistic informal interviewing with mothers.

Observations involved everyone who attended the NICUs. For interviews however, 17 mothers of various ages, parity, levels of education and previous breastfeeding experiences were invited and consented to be interviewed. Data collection continued until a saturation level was achieved, that was when a redundancy of emergent issues occurred and no new information was gained (Morse 1991).

The researcher visited the wards intermittently and at different times during the day which made it possible to obtain a broader range of data. Each period of observation lasted for four to six hours and was conducted at different times and on varying days of the week over the study period. The researcher did not undertake fieldwork during night shifts, because visitors, including mothers, were not allowed to visit at this time, and because staff members work shifts, which gave me the chance to meet them all during normal fieldwork hours. Observations focused on mothers' interactions with their infants, nurses' interactions with mothers and families, any breastfeeding or breast pumping activities, and other daily nursing activities.

Contemporaneous written notes were taken as far as possible. In cases where this was not possible, brief notes were made as an 'aide memorie', and then detailed notes written up as soon as possible afterwards.

\subsection{Interviewing}

In addition to fieldwork observations it was very important to know how the mothers and the health care team viewed breastfeeding preterm infants within the context of the neonatal intensive care wards. Qualitative interviewing was thus selected as part of the data collection methods.

My interviews were semi-structured. The researcher's position reflected Morse and Field's (1996) suggestion that semi-structured interviews are appropriate when the researcher has an idea about the research questions but not the answers. Any empty room within the NICU units was used at a convenient time for the participant.

The interview consisted of a two way conversation in which the researcher followed the conversational threads opened up by the interviewee (Polkinghorne 2005, p. 142). After doing some interviews, starting with a warming up question like "tell me about your pregnancy" was found a good way to start the interviews. A guideline was used to direct the interviews, which was informed by the initial analysis of the previous interviews. This enabled for exploration of issues previously identified. Additional questions were then asked depending on the flow of the interview, which were followed by additional probing. Probes were used where more clarification was needed. Any non-verbal reactions e.g. facial expressions and body language were then written as part of field notes. 


\subsection{Research setting}

Two NICUs in the North of Jordan were selected for multiple reasons. Firstly, there is little qualitative research that has been undertaken in the North of Jordan. The second reason was that the two units are located within the two largest hospitals in Irbid governorate, which has the second largest population and the highest population density in the kingdom. Finally, they are among the main level III referral units in Jordan.

\subsection{Ethical considerations}

Fieldwork began with gaining ethical approval from the Faculty of Health, Social Care and Education Research Ethics Panel (FREP) at Anglia Ruskin University/ UK. Once gained, ethical approval was then sought from the Jordanian Ministry of Health (MOH) for the public hospital and the university hospital's institutional review board (IRB). Both granted their specific ethical approval.

\subsection{Data analysis}

Preliminary analysis of the data during the data collection stage was important as it allowed to identify gaps that required further exploration before leaving the research context.

All interviews and observational data were transcribed to form the material for analysis. Although transcribed, voice recordings stayed as part of data to be analysed because repeated listening to tapes is often a "neglected area of analysis and yet one of the most essential" (Skeggs 1994, p. 84). Data analysis began with listening to the voice recordings and repeatedly reading the field notes and transcripts in order to identify categories and patterns. Richness of understanding is achieved through an analysis of not only the words but also the tone in which they were spoken.

\section{Results}

\subsection{Observations of the units}

At the time of commencing this study, the two research settings provided care for both preterm and full term infants with any medical complications. Within the two units, infants were fed according to a schedule feeding, usually every $2-3$ hours, and by bottle. Two types of formula were given: Similac Advance a preprepared infant formula and Similac Neosure formula for preterm and low birth weight infants. At the time of the study, only site 2 had WHO/UNICEF Baby Friendly Initiative accreditation. It supported exclusive breastfeeding in its postpartum unit and forbad the use of bottles and formula within these units. In site 1, however, bottles were allowed on the postnatal wards if requested by the mothers. In the case of normal delivery, both hospitals taught mothers about breastfeeding and provided support after delivery. In the postpartum units in both hospitals, there were many wall posters that discussed the benefits and other issues regarding breastfeeding. However, within the neonatal units, it was different. There were no posters, no handouts explaining the benefits of breastfeeding and no pumping facilities, the use of formula and bottles was considered totally acceptable, indeed was the norm. Within the two neonatal units, ready-to-use infants' formulas were often provided free of charge by various drug companies.

Throughout the entire fieldwork, the researcher witnessed only 2 pumping activities in site 1 and none in site 2 . Both mothers used an old style manual pumping machine brought by the mothers themselves, and they used privacy screens around them to carry out the pumping; in both cases, the mothers were left on their own without any assistance.

Besides lacking pumping and storing facilities, there was no private space for breastfeeding, no convenient place around the infant incubator for the mother to stay, and skin-to-skin contact was never encouraged between mothers and their infants. Along with, the high noise levels, especially in site 2 , these factors were all potential barriers to promoting and facilitating breastfeeding and a family centred approach. Mothers' presence in the NICUs varied during the day. Those staying in the postnatal ward usually visited their infants more often, and may visit during the night shift. Those coming from their home generally visited once a day, although some only visited once every few days, depending on factors such as distance to hospital, travel costs, other family responsibilities, and their husband's work. The visiting parent, mainly the mother, usually sat beside the infant's incubator on a regular chair (leather or plastic in site 1 and plastic on site 2) if available, which was uncomfortable for breastfeeding purposes. During the visit, mothers were not allowed to take their infants out of the incubator without permission. In site 2 , visiting was limited to parents only; this contrasted with site 1 where parents and sometimes grandparents were allowed to visit. In both units, visits are only permitted during the day and all visitors to both units are encouraged, via posters that hang over each sink, to wash their hands or use medical gel prior to their entry; however, hand washing was not strictly monitored.

Although there was recognition of the need to involve mothers and to communicate effectively with them by the staff members, the actual involvement of the mothers in their infant's care was minimum in site 1 and nonexistent in site 2. Minimal interactions were observed between the nurses and the parents. These interactions were usually initiated by the parents and were limited to asking a clarification question, commonly about the infant's condition and feeding. The interaction generally ended once the question had been answered. No active breastfeeding assistance was seen during the entire fieldwork period. The presence of fathers in the unit was rare and when observed they were seldom engaged in any activities. They usually took the lead for asking questions about their infant's health status especially when the doctor was around. However, answering their questions was not always welcomed by many of the staff members

The two units lacked facilities to accommodate the whole family or even the mothers. Waiting areas were provided outside the units, but longer stays were not possible under the current situations. Only a few chairs were provided in the units for the use of mothers while breastfeeding. As site 1 was a large unit, it could be rearranged to accommodate a seating area for the family. However, in site 2 this was not possible due to the small physical size of the unit and demands on the limited space.

Observation revealed that most of the space within the two units was taken up with the high technology equipment, which created a barrier to implement a family-centred care approach. Family involvement was severely restricted in both units and was only limited to the mothers. In the current situation, both units limited the mothers' abilities to care for their infants and to practice breastfeeding, and staff focused more on the technical and medical aspects of the infants' care. However, a greater involvement of the mothers on their infant's care plan would probably enhance the mother-infant attachment process, promote breastfeeding, improve mothers' emotional status (Kearvell \& Grant, 2010) and result in less workload for the staff.

\subsection{The mothers' perspectives}

Seventeen mothers of various ages, parity, levels of education and previous breastfeeding experiences were interviewed. All mothers, except one $(n=16)$ were Muslims. Maternal age ranged from 22 to 41 , with an average age of 29.7 years. All mothers were married $(n=17)$ and the majority had caesarean section deliveries $(n=11)$. Nine of them were college or university graduates, while the rest $(n=7)$ had a primary or secondary school education. The majority were unemployed $(\mathrm{n}=13)$, low to middle social class, and primiparous $(n=12)$. Two mothers gave birth to twins that made the total number of preterm infants 19 . They were born with a gestational age between 29 and 36 weeks with a mean gestational age of 33 weeks. Generally, the mothers at research setting one had a higher level of education and were of a higher social class than the mothers at research setting two. 
The mothers' stories begin with an unexpected change in the normal flow of their pregnancy and ends with the birth of a preterm baby in the NICU. Suddenly, they realized that mothering and breastfeeding would be relatively different experiences from what they had expected. Breastfeeding became a complex process for some and impossible for others. Four distinct themes emerged from the mothers narratives: the crisis, the control struggle, the separation, and becoming a mother. Almost all the mothers in this study spoke about going through all these stages during their infants' stay in the NICUs; however, each mother experienced each stage differently according to her previous experience and the amount of support that she had.

\subsubsection{The crisis}

The mothers participating in this study considered the birth of a preterm infant a crisis that caused great stress. Most of the mothers were not prepared for a preterm birth, thus the neonatal admission was stressful. Many elements were identified that represent being in a crisis including emotional instability, choosing not to visit the baby, infant's shocking appearance, feeling guilty and finding the NICU a stressful environment.

All of the mothers $(n=17)$ in this study reported being stressed and responding negatively to the birth of their baby as a result of the sudden change in their pregnancy and their infants' admission to a critical care unit. They described their experiences of having a preterm infant in the NICU as full of strains. A lot of stress was reported as being related to the infant's health status and survival, the environment of NICU, the staff attitudes, their abilities to perform maternal activities and to their identity as mothers. Feelings of sorrow and fear dominated the mothers' narratives:

At that night I felt like I was caught in a fire, it was heartbreaking. I was terrified of losing the baby. I did not worry about myself at all, but about my baby.

Guilty feelings were also reported and were identified as a way that parents used to adapt to this crisis:

When I came for the first time, I looked at him but I didn't cry. I don't know what my feeling was at that time? I can't describe those feelings. I stood up in front of his incubator and spoke to him. I told him: I don't know what to do for you, and I don't know if I was the cause of what is happening to you!

Some mothers used a strategy of not visiting their infants during their initial admission to the NICU in an attempt to lessen the loss if their baby died.

At the beginning I refused to see him. When I was in the delivery room I told them [nurses] that I do not want to see him, I was worried about losing him, may God forbid. I did not want to get attached to him that's why I refused to see him.

Mothers reported that the NICU environment generated a stressful reaction because they felt scared of the surrounding environment The initial visit to the NICU and the sight of the medical equipment and machines was shocking for many of them $(n=11)$. The fear of the high-tech neonatal environment added much to the mothers' negative feelings of having a preterm infant.

I was terrified he was connected to many devices, which scared me. The monitors' alarms scared me too. The alarm sounds became part of my dreams at night. I could hear them while sleeping.

The study findings also showed that mothers of preterm infants experienced overwhelming and different levels of anxiety, stress, sadness, fear, guilt, anger and oppression within the NICUs.

\subsubsection{The control power}

The second main category is the control power in which control relationships existed between staff members and mothers. Many forms of power relationships were observed including lacking control and needing to seek permission from the nurses, using a foreign language and giving mothers a subordinate role. The results identified a controlling power that placed mothers in a subordinate position to the nurses. The following is an example from field notes:
One mother came to ask about her baby. She greeted the nurses, who were sitting at the nursing station and were busy with paperwork, but she received no answer. She asked for permission to see her baby but was told that she could only look at him through the window. (Fieldnotes, public hospital. Mon.18.4.2011)

The mothers' narratives and actions demonstrated a lack of control as they talked about situations where nurses controlled their interactions with their infants. For example, some mothers described how they had to seek permission to visit and interact with their infants. On other occasions, they gave examples of being very stressed while waiting for the "appropriate time" to get access to the NICU:

This time I was so tired I could not leave the bed for two days. When I got out of bed I immediately came to visit him, but she [the nurse] refused to let me in, because, according to her, it wasn't appropriate time for the visit because there was a medical round. On a few occasions, mothers were left feeling upset and oppressed by the nurses' action of control. The nurses' constant presence in the NICU may create a belief that they own the unit, which results in them excessively controlling the access mothers have to the unit. The following illustration from fieldnotes describes a situation in which the nurse was inconsiderate to the mother's feelings and her presence and was acting as the owner of the infant and the place:

A mother came to breastfeed her baby at the 11 am feed to find that the nurse had just bottle-fed him. She appeared frustrated. I learnt afterwards that she had come early that morning for the 8:30 meal to find that he just been fed. She chose to wait outside for the 11am feed. The nurse forgot that the baby's mother had arranged with her to come and feed her baby, and then she forgot again about the mother's presence at the 11 am feed. The mother, with tremendous patience, expressed her willingness to wait again for the next feed 3 hours later. I felt sorry for her and told her that she could ask to wait inside the unit at her baby's bedside instead of waiting outside. She was pleased to know that this was possible. When she asked, the nurse did not approve her request, but told her that she would give her the baby so she could spend some time with him in the breastfeeding room. (Fieldnotes, public hospital. Sun.27.2.2011).

One mother described how she experienced a lack of control and increased anxiety when the health team were discussing her daughter's condition in English and she could not understand what they were saying. She wanted to know what is going on:

They [staff] were using English language in their discussions and I did not understand what they were saying. Even though I have a university degree in English language but I did not understand what they were saying, because they were using medical terms that I did not understand. They were debating about the existence of something and how to solve the problem. They said that her hand, I do not know what, and that her breathing, I do not know what, and because I did not understand, my fears have multiplied. The use of jargon and medical terminology during the medical rounds was another feature that made parents feel out of control over their infants' situation. In such cases, mothers commonly felt uncertain and unaware of their infants' situation because again they could not understand the medical team's discussion. In the previous quote for example, the mother thought that the staff were hiding something about her infant's health status, which intensified her feelings of anxiety.

\subsubsection{The separation}

The third main category derived was separation. During this phase, mothers reported many negative emotions that arose because of separation from their infants. The following elements were identified in this category: difficult to accept, feeling like stranger and not being important and physical distance.

Most of the participants described how difficult it was to be discharged from the hospital without having their babies with them: After I gave birth, I had to leave the hospital without her [the baby]. This increased my sense of anger and I was asking myself: 
with all this waiting and suffering, why have I need to leave without her? That increased my pain and suffering, I felt emotionally tired.

During their infant's hospital stay, mothers reported that they felt like strangers when they were separated from their infants.

I didn't feel that they belong to me. You know, I feel that it is just a dream; I didn't feel that I had given birth, and I didn't feel like a mother.

Being unable to breastfeed or offer breast milk made some mothers feel their infants did not need them. Additionally, and depending on which part of the NICU they were in, some mothers did not have a place to stay with their infants. This separation between the mothers and their preterm infants and the inability of mothers to spend as much time as they wanted with their babies signaled an infant focused care, in which mothers were left feeling unwelcomed and unimportant.

The visiting policy accelerated the mothers' feeling of being just a visitor. Even though site 1 visiting policy allowed mothers to visit any time, nurses restricted the visit for certain hours during the day, resulting in limited interaction between mothers and their infants.

I feel that my presence here in not important for him. What am I coming to do? The other reason is the unit here, you stay standing up all the time, there is no place to sit down in this room, and you feel like you are not welcomed.

Breastfeeding was a motivating factor for the mothers to frequently visit and interact with their infants. In addition, it contributed for the mothers' feeling of being important:

If breastfeeding was allowed I certainly would come every day.

The above extracts suggest that the quality of interaction between mothers and their infants, as well as their feelings of being important, were determining factors in the frequency of their visits. They needed to be acknowledged and to be part of their infants care plan in order to make sense of their presence. The need for physical closeness was strongly identified in the mothers' narratives as important in order to feel like a mother.

I wish if I could have a place available at the unit to stay with him all the daylong, as you see, I am only allowed to stay with him for the breastfeeding, and when I am done, I have to wait outside.

Feelings of sorrow and sadness dominated the mothers' narratives because of the physical distance between them and their infants. Mothers experienced enormous distress when separated from their preterm infants

\subsubsection{Becoming a mother}

The final category that emerged from the data analysis was "becoming a mother", in which mothers described many elements that made motherhood real for them. This category involves: the special moments, breastfeeding as a turning point and practical and informational needs.

Many of the participating mothers initially reported they did not feel like mothers and described different points, after giving birth, where they started to feel like real mothers. For the first time mothers, the phrase "becoming a mother" dominated when they talked about the first time that they felt like a real mother. For mothers who already had children this issue was not a concern, however, they talked about the first time when they had more control of the infant and when they felt that the baby became theirs.

Mothers remembered vividly the moments when they felt like mothers. The first visit, the first time they held their baby and the first breastfeed were significant moments for the mothers that made motherhood more real. The frequency of their visits was linked to the process of transformation into motherhood. Some mothers suggested that they initially felt detached from their in fants but after a few visits they began having different feelings and started to feel that the baby belonged to them:

When I visited him for the first time, I had a strange feeling which I can't describe, may be because I didn't touch him nor hold him.
But when I visited him for the second time, it was different; I felt that he is my baby then.

Mothers described the experience of holding and touching their babies for the first time as a significant moment that appeared to make motherhood more real. Touching and holding a baby is usually the first step toward breastfeeding. One mother described how holding her baby for the first time gave her warm feelings that made her want to breastfeed him even though she had no breast milk:

In my last visit, they took him out of the incubator and I cuddled him. It was the first time to touch him, which was 20 days after he was born. [Mother's voice began trembling as she talks] I put him in my lap, and tried to breastfeed him despite the fact that I don't have milk anymore and he was not hungry because he had already finished his bottle feed. Even though, I felt affectionate towards him. I felt that he is mine, he became my own, he is my first baby. The participants identified breastfeeding as the most important turning point for their experience of becoming a mother. This was evident in their narratives:

For me, feeling like a mother has started with the first breastfeed. Breastfeeding was a cultural norm for all of the study participants. All mothers $(n=17)$ in this study planned to breastfeed their infants for at least the first six months. Of the 17 participants, 14 mothers expressed breast milk in order to maintain their milk supply. The other 3 did not express because they were not told this was possible. Of the 14 who expressed, 9 discarded their milk because they did not know about the possibility of storing it. Only 5 provided milk for their infants, 3 of whom were advised to do so by the staff. Of the 14 mothers who expressed milk, 7 succeeded in breastfeeding later, 9 were still waiting for their infants' conditions to improve before being allowed to breastfeed and one mother's milk dried up within the first two weeks. For all the mothers who succeeded, breastfeeding was a distinct point in becoming a mother, particularly for the first time mothers.

I continued crying until yesterday when my baby breastfed from me. The moment I started breastfeeding, I felt relieved. It was only then when I felt that I have become a real mother.

Another mother described how her maternal feelings started and developed over time, but it was with breastfeeding when it increased and motherhood became real:

When I gave birth, my feeling of happiness has increased and I experienced the beautiful motherhood feelings. These feelings have increased and became real when I breastfed him, I felt at that time I actually become a mother [smiling].

Mothers talked with joy about their first experience in breastfeeding and how it was nothing like anything they had ever felt before, it was an "indescribable" event according to them:

This is the first time for me to breastfeed; it was only yesterday when they allowed me to hold him. When I put him on my chest I felt like a mother. I felt nothing like this before.

What I care about now is that he started breastfeeding from me. This was a relief for me. The feeling was indescribable. It was only then when I felt that I became a mother.

The mothers' milk itself is connected to the transformation into a mother. Mothers referred to "my milk" in a way that highlights its importance and significance for their infants' health:

I felt so happy when they increased the amount of his feeds from my expressed milk. I felt so happy because I am seeing him improving and I felt that it is because of my milk.

Despite the powerful connection between breast milk and becoming a mother, most of the mothers in this study did not express their milk immediately after the birth of their babies. That was because, for some, their main concern had shifted toward the health of the baby, and many were unaware that they could give their milk to their infants. The medical staff did not encourage early initiation of expressing breast milk and mothers who decided to use a pump were mostly encouraged to do so by family members:

At the beginning my breasts were engorged and tender to touch. When I asked them here about breastfeeding, they answered that during their first period of life, preterm babies can't tolerate 
breastfeed. And I asked them again by phone and they told me that he still having milk through a tube. So my milk starts diminishing by time until it ceased. Nobody here told me what to do to maintain my milk production. My mother and my aunt told me to pump it. I did for a while, but I don't have milk anymore. I tried to pump but had nothing...every time I asked to come and breastfeed, the answer was not yet, still on the feeding tube.

All the mothers in this study said that they were delighted to become mothers. However, they went through different levels of anxiety. Some were faced with the fear of the unknown in terms of the responsibilities for the new arrival. Mothers wanted the unit to be a learning environment to ease their fears and to increase their self-confidence. They wanted a more cooperative relationship with the nurses, to learn from them about how best to deal with their infants at home. Confidence in looking after preterm infants at home is an important aspect to becoming a mother. Some mothers talked about being frightened of taking their infants home because they did not feel confident in looking after them:

I am frightened of the idea that I will go home with both of them. I don't know how I am going to deal with them.

\section{Discussion}

The transition into motherhood is a key concept for this study that will be developed in this section. This study has shown that the transition into motherhood for mothers of preterm infants was not what they expected. They missed the joy of having a full term healthy baby; instead, they had to deal with a crisis filled with stress and anxiety. Mothers had periods of vulnerability and adjustment throughout their transition to motherhood. These findings were not unique for the Jordanian mothers; similar findings have been identified in many other cultures. For example, Hutchinson et al. (2012) interviewed 12 preterm infants' parents from diverse racial backgrounds with the majority being Caucasian. They developed a model of parental progression during their infants' transition from the NICU to home, in which parents proceed through four phases from not being a parent to being a parent.

In this study, the mothers' transition into motherhood was not without psychological distress as they experienced feelings of powerlessness, a lack of control, not belonging, disorientation and being neglected. They experienced an unexpected change in their pregnancy path that led to their infant being admitted to the NICU. The birth of a preterm infant itself is a crisis that places a great strain on the mother's role et al.1999; Lindberg \& Öhrling 2008). Mothers are not prepared for becoming a mother because everything happens quickly and unexpectedly, and they lose the anticipated delivery of a full term healthy infant (Stern \& BruschweilerStern 1999). Mothers may experience a sense of failure because unable to complete the pregnancy (Griffin et al. 1998). They are unable to perform their role as mothers because of the unexpected early birth and their infant's vulnerability. Many mothers experience uncertainty about their role as a mother and of their infants' health status (Holditch-Davis \& Miles 2000).

The threshold of motherhood is one of many that most women cross at some point during their lives. Some of these thresholds are connected with biological events such as life and death (Teather 1999). Others are associated with social development such as adolescence or a change in economic role such as retirement. Motherhood is a rite of passage, a concept originating in the work of the French anthropologist Arnold Van Gennep (1873 1957). It is about a transformation process, a turning point in a person's life where they move from one state to another and enter a new status group. Van Gennep defined rites of passage as "rites which accompany every change of place, state, social position and age" (as cited in Turner 1967, p.94).

A rite of passage consists of three phases, where each has its associated rituals: the separation or detachment rites (preliminal), the transition rites (liminal), and the incorporation rites (postliminal) (Van Gennep 1960). The second stage of liminality is the one that has the greatest analytical importance for this research. The term liminal relates to a transitional or initial stage of a process or, occupying position at, or on both sides of a boundary or threshold (OED). Liminality is a phase of ambiguity and uncertainty, of being "betwixt and between", the person no longer belongs to the old state and not yet to the new (Turner 1987). During this liminal period, Turner described the subject of passage ritual as "invisible". The "transitional-being" or "liminal persona" is "at once no longer classified and not yet classified....neither one thing nor another neither here nor there; or may even be nowhere" (Turner 1987, pp. 95-97). A person in this state often feels isolated or disconnected from the before and after states (Turner 1987), and this creates feelings of inadequacy and uncertainty which arise from the feeling of not fitting in or not belonging (Wendling 2008).

Pregnancy and childbirth are transitional periods for women. The beginning of the pregnancy is the starting point for rites of passage into motherhood. A pregnant woman at this point separates herself from her formal social identity. The length of this separation phase depends on the time required to confirm the pregnancy status and on the pregnant woman to fully accept her pregnancy (DavisFloyd 1992).

Childbirth is "intended to reintegrate the woman into the groups to which she previously belongs [in the case of multiparous] or to establish her new position in society as a mother" (Van Gennep 1960 , p. 41). Giving birth is a transition that many women go through and normally transforms women into mothers in a very predictable way. During the periods of pregnancy, childbirth and the immediate postpartum period, the woman is in the transitional liminal phase. At this stage, cultural transformation may overlay the pregnant woman's experience of the physiological process of becoming a mother (Davis-Floyd 1992).

In Islamic cultures, such as Jordan, there are shared rites and rituals associated with pregnancy and childbirth that are directed towards having both a healthy mother and a healthy baby. These rituals are also seen as spiritual acts. Women get a lot of attention and support from their family members during pregnancy and the postpartum period. Generally, there is help with the housework and a desire to ensure the mother has a healthy diet. During these periods, ingestion of any harmful substances, such as cigarette smoke, is forbidden.

Although it is much shorter in terms of time when compared to the pregnancy period, the birth process itself is a very important rite of passage to becoming a mother. Childbirth "brings each mother into a dramatic and sometimes distressing encounter with her own body as it undergoes astonishing changes, in a matter of minutes passing through the metamorphosis of delivery to experience an empty body" (Kitzinger 1987, p. 172).

Breastfeeding is an important ritual in raising a baby, and is considered the norm in Islamic countries; it is recommended by the Quran that breastfeeding continue for two years. Islam also values the breastfeeding relationship between mother and baby and in cases where the biological mother cannot breastfeed her baby a wet nurse is employed. The wet nurse in Islam is considered a mother and thus her biological children become the baby's brothers and sisters and her husband becomes a father; future marriages between the two families is forbidden. This signifies the level of importance that Islam has for a mother's milk.

Women need to become mothers not only because they are biologically capable of doing so, but also because of the many social and cultural expectations and a lack of other opportunities (Oakley 1974; Khalaf \& Callister 1997). This is true in Arab cultures where having children is the primary motivation for marriage. Jordanian women experience personal, social and marital adversities when they are diagnosed as infertile (Obeisat et al. 2012). They are socially stigmatized as being useless, and people may consider the infertile woman "a tree without crops, which must be cut off from roots" (Obeisat et al. 2012, p.11). Moreover, one of the most important arguments for a man to practice polygyny and have a second wife is if his first wife is infertile, which makes Jordanian women anxious about having children.

Being acknowledged as a mother is very important for Muslim women because mothers have the highest order of human relation- 
ships in Islam. Allah describes in the Qur'an how parents should be treated. He refers to the recognition of one's parents after the recognition of Allah himself:

Thy Lord hath decreed that ye worship none but Him, and that ye be kind to parents. Whether one or both of them attain old age in thy life, say not to them a word of contempt, nor repel them, but address them in terms of honour. And, out of kindness, lower to them the wing of humility, and say: "My Lord! Bestow on them Thy mercy even as they cherished me in childhood" (Qur'an: Al Isra, 17: 23-24).

Mothers are given a higher status in the Qur'an because of their suffering and hardship in their role as mothers.

And we have enjoined on man (to be good) to his parents: in travail upon travail did his mother bear him. And in year twain was his weaning: (hear the command), "show gratitude to me and to thy parents: to me is (thy final) goal (Qur'an: Luqman, 31: 14).

This hardship was even deeper and more intense with the women in this study, where most of them went through complicated pregnancies that led to the preterm birth.

In most cases passing through the rites of passage into motherhood occurs in a very predictable way. The very predictability of this transformation is precisely what generates a mother's stress when the predictable becomes the unpredictable. Giving birth to a preterm infant is a deviation from what is expected at the beginning of a new emotional period. The difference between giving birth to a full term versus a preterm infant might be the duration the mother spends in the liminal space. In the case of full term, normal births mothers cross the threshold into motherhood very quickly however, for mothers of preterm infants being held in the liminal period often creates a sense of vulnerability. The duration of this liminal period varies according to the infants' condition, the duration of infant's hospitalization and the degree of the mothers' participation in the care of the infant. A state of ambiguity and uncertainty regarding their role and their infants' health during this phase was clearly experienced by most women.

Liminal individuals share common emotional experiences (Turner 1967). Women in this study shared many components of being in a liminal phase, such as powerlessness, lack of control, being detached and ignored. Women reported that they continually felt confused and detached from being mothers. They were placed in liminal positions because of these components, and were inhibited from progressing into motherhood by the health care system within the neonatal units. What the theory "rites of passage" failed to address therefore, is what happens when a person in the liminal phase cannot move on but is held in this phase. The women in this study were starting their transformational passage into motherhood, but instead of passing through they were held, indeed encouraged to be even deeper, into their liminal phase. I refer to this as being 'suspended in liminality'. Women are suspended in a liminal position until their infant's health status improves, and then they are able to move on and become transformed into mothers.

Maternal feelings started in pregnancy but were placed in a state of suspension after birth because of the infants' critical condition The admission of a newborn baby to the NICU causes disruption in the mothers' transition to motherhood, thus delaying their identity recognition (Zabielski 1994; Nelson 2003). Feelings of physical separation voiced by the mothers and the restricted contacts between the mothers and their infants further suspended mothers in their liminal positions and delay the formation of their identity as mothers, and thus prolonging their in-between status. This separation prevented mothers from achieving many of their expected motherhood experiences such as caring, cuddling, touching and breastfeeding. Caring in general and breastfeeding in particular are important rites of passage into motherhood, not only for preterm infants' mothers but also for all mothers. Therefore, hindering mothers from participating in their infants' care and from breastfeeding will have a negative impact on establishing the women's new identity of becoming a mother. For a mother to establish a maternal identity is very important as it contributes to a women's psychosocial development, leading to an increase in her adaptive behaviours (Mercer 2004).

The use of English by the health care professionals during the medical rounds adds to the mothers' experience of being in a liminal space as it provokes feelings of uncertainty and disorientation. All mothers in this study spoke Arabic, with some having limited English skills. More significantly, mothers were not able to understand the health team's English which contained many medical terms. This made mothers feel disorientated and uncertain as they were not engaged, which caused a lack of awareness regarding their infant's care plan. The use of medical language generally is a source of problems and poor communication for many patients (Bourhis, et al.1989), and can easily lead to incorrect interpretations of information given, which may have an adverse effect on patient health. In fact, the US Centre of Disease Control reported that nine out of ten adults have difficulty following medical advice because it is incomprehensible to the average person (Landro 2010).

English, as the dominant global language, is considered the language of power and professionalism for Jordanians. Therefore, for many Jordanians anyone speaking English is regarded as prestigious and knowledgeable. Bearing this in mind, speaking English in the mothers' presence may build the self-esteem and selfsatisfaction of the medical health care team, but at the same time, it may also intensify the mothers' feelings of powerlessness and uncertainty and distance the mothers from the health care team. In this study, the mothers were seen to interpret the use of English by the health care team in front of them as an attempt to hide unpleasant information about their children's health, which accelerated their anxieties and feeling marginalized, heightened their suspension in their liminal position.

Whilst this study identified how the NICU environment and the nurses' behaviours contributed towards holding women in a liminal position by inhibiting their caring for their baby, it has been recognized that the act of breastfeed itself may also be viewed as maintaining mothers in a liminal state (Sachs 2005). Breastfeeding positions women in liminal states as they "struggle to keep their milk, their breasts and their relationships with their babies in their socially ordained right place, well out of sight" (Kirkham 2007, p. 2 ), in recognition of the potential for social embarrassment when breastfeeding in public place (Condon et al. 2013), such as the NICU. Additionally, breastfeeding women may have to modify their diet and alter their habits to ensure the suitability of their milk (Sachs 2005), all of which may enhance their experience of liminality.

The use of liminality as a framework to understand the experience of breastfeeding was first introduced by Davis-Floyd and Sargent (1997), and further elaborated by Mahon-Daly and Andrews (2002). Breastfeeding mothers experience liminality as the boundaries between themselves and their infants remain blurred (Schmied \& Lupton 2001). Breastfeeding marginalises women from their everyday life. Sachs (2005) described breastfeeding as a life passage, which could be visualised as a literal passage, with the choice of opening two doors at the birth of the baby; bottle feed or breastfeed.

The liminality considered in the studies of Mahon-Daly and Andrews (2002) and Sachs (2005) related to women whose passage from womanhood into motherhood was clearly recognized by the presence of the baby and care provision by the mother. This movement and recognition, both personal and public, had not yet occurred for the women observed and interviewed in this study. Mothers whose preterm babies were being cared for in NICUs were seen to be held in a state of suspension, with minimal of any acknowledgement of motherhood, despite having delivered a baby. Their contribution towards the nourishment of their baby was also denied. Unfortunately, both issues hold the potential for detrimental effects on maternal and infant wellbeing.

Breastfeeding is a deep human experience which encompasses mothers' perceptions of themselves as women and mothers, and therefore their relationship with their infants and family (Spencer 2008). When this is desired but not 'allowed' or supported in the 
NICU situation, the potential for serious and fundamental maternal distress becomes apparent. In such situations, mothers may develop a variety of coping mechanisms to assist them through this difficult passage. In this study, it became apparent that many of the mothers found comfort through their religious beliefs and practices. Whilst this may be specific to Jordanian or Moslem societies, it highlights the importance religion holds for some people living through difficult situations, suggesting this is worthy of recognition and understanding by staff caring for such individuals. During difficult situations and periods of uncertainty, spiritual and religious perspectives can provide a context where anxieties and stress may be faced (Yuen 2011). During the stage of transition, it is often considered that a person comes to believe in the protective powers of the divine (Turner, 1969). Almost all the participating mothers expressed a sense of spiritual awareness, a force or a higher power beyond their control that helped them to cope with their fears and anxieties. Some research reifies spirituality and religiosity as important components of health and well-being for many (Baumiller 2002; Callister \& Khalaf, 2010; Yuen 2011). Pregnancy and childbirth have been particularly perceived as spiritual events throughout history and across cultures because of the miraculous processes involved (Obeidat \& Callister 2011; Linhares 2012). Callister and Khalaf (2010) identify childbirth as a time to get closer to God and the use of religious beliefs and practices as powerful coping mechanisms. In the current study, issues of faith dominated the mothers' narratives. Mothers regarded what happened to them as the will of Allah. During this vulnerable time, "connecting with a spiritual source for insight is essential to activate the deeper powers of a rite of passage," (Wendling 2008, p. 3) and, for many mothers, to reduce their fears of the unknown. Mothers concerns were lessened by the belief that everything in their lives was pre-planned by Allah, and that $\mathrm{He}$, the most gracious and most merciful, is taking care of their infants.

The final phase of rites of passage is the incorporation phase, the rites of childbirth deemed to reintegrate women into the groups to which they previously belong as mothers, or to generate their new identity as new mothers (Van Gennep 1960). The experience of having a preterm infant in the NICUs delayed incorporation and affected mothers differently. For first-time mothers, the rites of passage were most acute, as mothers were facing the challenges without previous experiences. This passage is also a major transition in the women's roles; it is moving from one status into another, a more honoured one. A woman is becoming a mother, and this can only happen once. For experienced mothers, however, it is a matter of extending the family and accommodating their preterm infants into this family. Their transition is from mother to mother, which is an enhancement of an already gained status. For both, first-time mothers and experienced mothers, during the integration phase, liminality is replaced by moving into the new identity and/or having confidence in their mothering roles with the preterm infants. This phase comprises the true feeling of and acting like a mother.

What might be gained at the end of this transition process however may be quite different than what was initially expected (Wendling 2008). For some mothers of preterm infants, the transition ends with the infant's death, and thus the transition to motherhood will never be achieved with this particular pregnancy. For such women, the liminal stage may become a way of life rather than a transitional period, as they will never proceed to the next stage of what Van Gennep called postliminal or incorporation. Turner (1987) argues that some people may not move beyond this liminal space. In fact, these women will return back into their preliminal state, as just women. They are grieving for the loss of their infants, but also for their lost status. This situation is devastating for the first time pregnant women, because the long period of the pregnancy and the short period of being mothers is not acknowledged by their community.

The women's stories about their time in the two NICUs supported the argument that they experience being suspended within their liminal positions. This study argues that while these women were going through their transition into motherhood, their infants' ad- mission to the NICU held them in a liminal position. Being suspended in their liminality may have an impact on the mothers' psychological status and on their relationship with their infants. Nurses and midwives at the NICUs play an important role in helping mothers throughout their transition to motherhood. Making nurses and midwives aware of the different stages that mothers go through may develop a greater understanding of the mothers' needs while they are trying to adjust to their new roles as mothers in the stressful situation of having a preterm infant.

\section{Conclusion}

For the last few years, efforts to increase the awareness of Jordanian mothers' of the benefits of breastfeeding were seen to be successful (Mubaideen \& AL-Saraireh 2006). In this study, all the mothers were aware of the importance of breastfeeding and wanted to breastfeed. However, they faced many obstacles that prohibited them from practicing breastfeeding or other maternal activities such as cuddling or sometimes even touching their babies within the neonatal units. There was little evidence that mothers were assisted by the nurses to build meaningful relationships with their infants. Nurses were the primary carers of the infants doing even the simple activities that mother could do such as changing nappies. Under these conditions mothers did not feel themselves to be real mothers; rather they experienced feelings of being unimportant and unwelcome, feelings that placed them in suspended liminal positions because they had not yet become independent mothers.

This study argues that while these women were in going through their transition into motherhood, their infants' admission to the NICU held them in a liminal position. Being suspended in their liminality may have an impact on the mothers' psychological status and on their relationship with their infants. Nurses and midwives at the NICUs play an important role in helping mothers throughout their transition to motherhood. Making nurses and midwives aware of the different stages that mothers go through may develop a greater understanding of the mothers' needs while they are trying to adjust to their new roles as mothers in the stressful situation of having a preterm infant.

In order to overcome the crisis that mothers of preterm infants experience, it is important to help them make the transition into motherhood with minimal pain. The mothers themselves identified many things that helped them make the transition, including physical closeness with their babies, feeling important, needing to breastfeed or provide breast milk, participating in infant care and some practical and informational needs. Physical and emotional closeness have been identified as essential to the physical, emotional and social well-being of both the infants and their mothers (Flacking et al. 2012).

With appropriate support, a mother's role as the primary care giver can be restored while they navigate their way through the NICU's environment. Nurses within the NICUs play an important role in empowering mothers in order to facilitate bonding and attachment (Kenner \& McGrath 2004; Herbst \& Maree 2006).

\section{Acknowledgment}

The author is grateful to the mothers who participated in this study and to Dr Trudy Stevens for her constructive discussions and guidance, which helped me and informed my thinking all the way. Key messages

- For the mothers delivering prematurely, the rite of passage to becoming a mother is suspended in a liminal phase, which relates to their abilities to look after their babies within these units or once they are discharged.

- This study has identified breastfeeding and offering breast milk as central to the experience of becoming a mother.

- To feel like a real mother, mothers need to have unlimited access to their infants and to be empowered to take control and improve their experiences by breastfeeding their preterm in- 
fants. This is significant not only for the mothers' identity and psychological wellbeing, but also for the preterm infant's wellbeing.

\section{References}

[1] Ali, A.Y., 1989. The holy Qur'an: text translation and commentary. Amana Corp.

[2] Barclay, L., Everitt, L., Rogan, F., Schmied, V. and Wyllie, A., 1997. Becoming a mother--an analysis of women's experience of early motherhood. Journal of advanced nursing, 25(4), pp. 719-728. http://dx.doi.org/10.1046/j.1365-2648.1997.t01-1-1997025719.x.

[3] Baumiller, R., 2002. Spiritual development during a first pregnancy. International Journal of Childbirth Education, 17(3), p. 7.

[4] Bialoskurski, M.M., Cox, C.L. and Wiggins, R.D., 2002. The relationship between maternal needs and priorities in a neonatal intensive care environment. Journal of advanced nursing, 37(1), pp. 62-69. http://dx.doi.org/10.1046/j.1365-2648.2002.02057.x.

[5] Bourhis, R.Y., Roth, S. and Macqueen, G., 1989. Communication in the hospital setting: a survey of medical and everyday language use amongst patients, nurses and doctors. Social Science \& Medicine 28(4), pp. 339-346. http://dx.doi.org/10.1016/0277-9536(89)90035-X.

[6] Browne, J.V., 2003. New perspectives on premature infants and their parents. Zero to three, 24(2), pp. 4-12.

[7] Callister, L.C. and Khalaf, I., 2010. Spirituality in childbearing women. The Journal of perinatal education, 19(2), pp. 16 http://dx.doi.org/10.1624/105812410X495514.

[8] Condon, L., Rhodes, C., Warren, S., Withall, J., and Tapp, A., 2013. 'But is it a normal thing? 'Teenage mothers' experiences of breastfeeding promotion and support. Health Education Journal, 72(2), 156162. http://dx.doi.org/10.1177/0017896912437295.

[9] Davis-Floyd, R., 1992. Birth as an American rite of passage. Berkeley London: Berkeley London University of California Press.

[10]Davis-Floyd, R. E., \& Sargent, C. F. (Eds.), 1997. Childbirth and authoritative knowledge: Cross-cultural perspectives. University of California Pr.

[11]Fenwick, J., Barclay, L. and Schmied, V., 2001a. Struggling to moth er: a consequence of inhibitive nursing interactions in the neonata nursery. Journal of Perinatal \& Neonatal Nursing, 15(2), pp. 49-64. http://dx.doi.org/10.1097/00005237-200109000-00005.

[12]Flacking, R., Ewald, U., Nyqvist, K.H. and Starrin, B., 2006. Trustful bonds: A key to "becoming a mother" and to reciprocal breastfeeding. Stories of mothers of very preterm infants at a neonatal unit. Social science \& medicine, 62(1), pp. 70-80. http://dx.doi.org/10.1016/j.socscimed.2005.05.026.

[13]Flacking, R., Lehtonen, L., Thomson, G., Axelin, A., Ahlqvist, S. Moran, V.H., Ewald, U. and Dykes, F., 2012. Closeness and separation in neonatal intensive care. Acta Paediatrica (Oslo, Norway: 1992), 101(10), pp. 1032-7

[14]Gold, R.L., 1958. Roles in sociological field observations. Social Forces, 36(3), pp. 217-223. http://dx.doi.org/10.2307/2573808.

[15]Griffin, T., Wishba, C. and Kavanaugh, K., 1998. Nursing interventions to reduce stress in parents of hospitalized preterm infants. Jour nal of pediatric nursing, 13(5), pp. 290-295. http://dx.doi.org/10.1016/S0882-5963(98)80014-6.

[16]Hartmann, P.E. and Ramsay, D.T., 2005. Mammary anatomy and physiology. In: E. JONES and C. KING, Eds, Feeding and Nutrition in the Preterm Infant. London: Elsevier, pp. 53-68.

[17]Herbst, A. and Maree, C., 2006. Empowerment of parents in the neonatal intensive care unit by neonatal nurses. Health SA Gesondheid 11(3), pp. 3-13. http://dx.doi.org/10.4102/hsag.v11i3.232.

[18] Holditch-Davis, D. and Miles, M.S., 2000. Mothers' stories about their experiences in the neonatal intensive care unit. Neonatal Network, 19(3), pp. 13-21. http://dx.doi.org/10.1891/0730-0832.19.3.13.

[19]Hutchinson, S.W., Spillet, M.A. and Cronin, M., 2012. Parents' Experiences during their Infant's Transition from Neonatal Intensive Care Unit to Home: A Qualitative Study. The Qualitative Report, 17(23), pp. 1-20.

[20]Kavanaugh, K., Meier, P., Zimmermann, B. and Mead, L., 1997. The rewards outweigh the efforts: breastfeeding outcomes for mothers of preterm infants. Journal of Human Lactation, 13(1), pp. 15-21. http://dx.doi.org/10.1177/089033449701300111.

[21] Kearvell, H. and Grant, J., 2010. Getting connected: how nurses can support mother/infant attachment in the neonatal intensive care unit. Australian Journal of Advanced Nursing, 27(3), pp. 75-82.

[22]Kenner, C. and McGrath, J., 2004. Developmental care of newborns \& infants. Edited by Kenner C, McGrath JM.Mosby ed.St Louis MO, 63123 .
[23]Khalaf, I. and Callister, L.C., 1997. Cultural meanings of childbirth: Muslim women living in Jordan. Journal of Holistic Nursing, 15(4), pp. 373-388. http://dx.doi.org/10.1177/089801019701500405.

[24] Kirkham, M. (Ed.), 2007. Exploring the dirty side of women's health. Routledge.

[25] Kitzinger, S., 1987. The experience of breastfeeding. Penguin.

[26]Landro, L., 2010. Taking Medical jargon Out of Doctors' Visits. [Online]. Available at: <http://online.wsj.com/article/SB10001424052748703620604575349 110536435630.html\#articleTabs\%3Darticle>. [Accessed September 2012].

[27]Lindberg, B. and Öhrling, K., 2008. Experiences of having a prematurely born infant: from the perspective of mothers in northern Sweden. International journal of circumpolar health, 67(5), pp. 461-71. http://dx.doi.org/10.3402/ijch.v67i5.18353.

[28] Linhares, C.H., 2012. The Lived Experiences of Midwives with Spirituality in Childbirth: Mana from Heaven. JOURNAL OF MIDWIFERY \& WOMENS HEALTH; J.Midwifery Women Health, 57(2), pp. 165-171. http://dx.doi.org/10.1111/j.1542-2011.2011.00133.x.

[29]Lupton, D. and Fenwick, J., 2001. 'They've forgotten that I'm the mum': constructing and practising motherhood in special care nurseries. Social Science \& Medicine, 53(8), pp. 1011-1021. http://dx.doi.org/10.1016/S0277-9536(00)00396-8.

[30] Mahon-Daly, P. and Andrews, G.J., 2002. Liminality and breastfeeding: women negotiating space and two bodies. Health Place, 8(2), pp. 61-76. http://dx.doi.org/10.1016/S1353-8292(01)00026-0.

[31]McInnis, R. and Chambers, J., 2008. Supporting breastfeeding mothers: qualitative synthesis. Journal of Advanced Nursing, 62(4), pp. 407-427. http://dx.doi.org/10.1111/j.1365-2648.2008.04618.x.

[32] Mercer, R.T., 2004. Becoming a mother versus maternal role attainment. Journal of Nursing Scholarship, 36(3), pp. 226-232. http://dx.doi.org/10.1111/j.1547-5069.2004.04042.x.

[33] Morse, J.M., 1991. Strategies for Sampling. In: J.M. MORSE, ed, Qualitative Nursing Research: A Contemporary Dialogue. London: Sage Publications, pp. 127-145. http://dx.doi.org/10.4135/9781483349015.n16.

[34] Morse, J.M. and Field, P.A., 1996. Nursing research: the application of qualitative approaches. 2 edn. Thousand Oaks, Calif. London: Chapman and Hall. http://dx.doi.org/10.1007/978-1-4899-4471-9.

[35] Mubaideen, M. and Al-Saraireh, M. R., 2006. Breast-feeding pattern in selected antenatal care clinics in jordan. JRMS, 13(2), pp. 74-78.

[36]Nelson, A.M., 2003. Transition to motherhood. Journal of Obstetric, Gynecologic, \& Neonatal Nursing, 32(4), pp. 465-477. http://dx.doi.org/10.1177/0884217503255199.

[37] Oakley, A., 1974. The sociology of housework. Martin Robertson.

[38] Obeidat, H. and Callister, L., 2011. The lived experience of Jordanian mothers with a preterm infant in the neonatal intensive care unit. Journal of Neonatal-Perinatal Medicine, 4(2), pp. 137-145.

[39] Obeisat, S., Gharaibeh, M.K., Oweis, A. and Gharaibeh, H., 2012. Adversities of being infertile: the experience of Jordanian women. Fertility and sterility, 98(2), pp. 444-9. http://dx.doi.org/10.1016/j.fertnstert.2012.04.036.

[40]Polkinghorne, D.E., 2005. Language and meaning: data collection in qualitative research. Journal of Counseling Psychology, 52(2), pp. 137. http://dx.doi.org/10.1037/0022-0167.52.2.137.

[41]Redwood, T., 2007. Becoming a mother: a phenomenological exploration of transition to motherhood, its implications for the professional lives of nurses, midwives and health visitors, School of Nursing and Midwifery, University of East Anglia

[42]Roper, J.M. and Shapira, J., 2000. Ethnography in nursing research. Thousand Oaks, Calif. Sage Publications. http://dx.doi.org/10.4135/9781483328294.

[43] Sachs, A. M. (2005). "Following the line": an ethnographic study of the influence of routine baby weighing on breastfeeding women in a town in the Northwest of England (Doctoral dissertation, University of Central Lancashire).

[44]Schmied, V., \& Lupton, D., 2001. Blurring the boundaries: breastfeeding and maternal subjectivity. Sociology of Health \& Illness, 23(2), 234-250. http://dx.doi.org/10.1111/1467-9566.00249.

[45] Shakespeare, J., Blake, F. and Garcia, J., 2004. Breast-feeding difficulties experienced by women taking part in a qualitative interview study of postnatal depression. Midwifery, 20(3), pp. 251-260. http://dx.doi.org/10.1016/j.midw.2003.12.011.

[46] Skeggs, B., 1994. Situating the production of feminist ethnography. In: M. MAYNARD and J. PURVIS, Eds, Researching women's lives from a feminist perspective. Great Britain: Taylor \& Francis Ltd, pp. 72-92.

[47]Shin, H. and White-Traut, R., 2007. The conceptual structure of transition to motherhood in the neonatal intensive care unit. Journal of 
advanced nursing, 58(1), pp. 90-98. http://dx.doi.org/10.1111/j.13652648.2006.04194.x.

[48] Singer, L.T., Salvator, A., Guo, S., Collin, M., Lilien, L. and Baley, J., 1999. Maternal psychological distress and parenting stress after the birth of a very low-birth-weight infant. JAMA: Journal of the American Medical Association, 281(9), pp. 799-805. http://dx.doi.org/10.1001/jama.281.9.799.

[49] Spencer, R. L., 2008. Research methodologies to investigate the experience of breastfeeding: a discussion paper. International Journal of Nursing $\quad$ Studies, $45 \quad$ (12), $1823 \quad-\quad 1830$ http://dx.doi.org/10.1016/j.ijnurstu.2008.04.008.

[50]Stern, D.N. and Bruschweiler-STERN, N., 1999. The birth of a mother: how the motherhood experience changes you forever. New York: New York Basic Books.

[51]Teather, E.K., ed, 1999. Embodied Geographies: Spaces, Bodies and Rites of Passage. London: Routledge.

[52]Turner, V., 1967. Betwixt and Between: The Liminal Period in Rites de Passage. The forest of symbols: Aspects of Ndembu ritual. Cornell University Press, pp. 93-111.

[53]Turner, V., 1969-last update, Liminality and Communitas: Form and Attributes of Rites of Passage [Homepage of London: Aldine,], [Online]. Available at: 〈http://faculty.dwc.edu/wellman/Turner.htm> [Accessed August 2012].

[54]Turner, V., 1987. Betwixt and between: The liminal period in rites of passage. In: Mahdi, Foster and Little, ed, Betwixt and between: Patterns of masculine and feminine initiation,pp. 5-19.

[55]Van Gennep, A., 1960. The rites of passage. London: Routledge \& Kegan Paul.

[56]Watson, G., 2011. Parental liminality: a way of understanding the early experiences of parents who have a very preterm infant. Journal of Clinical Nursing, 20(9), pp. 1462-1471. http://dx.doi.org/10.1111/j.1365-2702.2010.03311.x.

[57]Wendling, G., 2008. Understanding Change through the Rites Of Passages Framework.

[58]Yuen, E., 2011. Spirituality and the Clinical Encounter. International Journal for Human Caring, 15(2), pp. 42-46.

[59]Zabielski MT, 1994. Recognition of maternal identity in preterm and fullterm mothers. Maternal-child nursing journal, 22(1), pp. 2-36. 